\title{
Torsten B. Moeller and Emil Reif (eds): Pocket Atlas of Sectional Anatomy, Computed Tomography and Magnetic Resonance Imaging, 4th edn. Vol. I: Head and Neck; Vol. II: Thorax, Heart, Abdomen and Pelvis
}

\author{
Thieme, Stuttgart 2014, ISBN: 978-3-13-125504-4 (Vol. I), 978-3-13-125604-1 (Vol. II)
}

\author{
Antonio Manna $\cdot$ Luigi Mansi
}

Published online: 3 March 2015

(C) Springer-Verlag Berlin Heidelberg 2015

We review here the 4th edition, revised and expanded, of two of the three books of a trilogy, also including a third companion volume on "Spine, Extremities, Joints", not recently revised.

The editors are Torsten B. Moeller and Emil Reif, who work in the Department of Radiology at the Marienhaus Klinikum Saarlouis-Dillingen in Germany. The support deriving from the collaboration with numerous professionals, such as radiology technologists, medical colleagues and the manufacturers Siemens and Philips, has been recognized.

This atlas has been and continues to be a great editorial international success, very popular worldwide. Both volumes are the authorized translation from the 3rd German Edition, edited in 2011 by the same publisher. Furthermore, both publications have been translated and edited, from previous editions of the last German version of 2011, in many languages, including French, Greek, Japanese, Korean, Portuguese-Brazilian, Turkish and Spanish. National editions of the second volume have also been published in Hungarian, Russian, Polish and Italian.

The major advancement with respect to the 3rd edition, dating back originally about 10 years ago, is mainly in the improvement allowed by new MRI images, largely obtained with 3 T scanners; with respect to CT, the availability of multidetector CT (MDCT), frequently at 64 slices, allowed the production of sagittal and coronal images, in addition to axial scans. In this way, many of the older figures have been replaced, with a significant editorial improvement. Furthermore, all efforts have been made in following the most recently established anatomical and clinical conventions in numbering and describing, for example, vessels and lymph nodes.

Recent technical advances in magnetic resonance imaging and in CT have allowed more definition that emerges effectively in these volumes. In particular, the best spatial resolution has naturally led to a greater distinction of the anatomical detail and to a better analysis, for instance of vascular and nerve components. In many cases, the didactic content has been further improved through magnification with additional captions. For example, implementing suggestions by actively critical readers of the previous edition, the spaces of the neck and the intracranial vascular region have been described with greater detail.

Despite the changes, the text preserves the style of previous editions, remaining as informative as compact and easy to use. The format remains based on two-page units, with highquality radiologic images on one side and brilliant full colour diagrams on the other. When needed, small locational diagrams are also provided.

In conclusion, we enthusiastically suggest these books to all professionals working in diagnostic imaging. Their value is very great either for medical students or for residents in nuclear medicine, radiology and radiotherapy.
A. Manna $\cdot$ L. Mansi $(\varangle)$

Second University of Naples, Naples, Italy

e-mail: luigi.mansi@unina.it 